\title{
The Third Vaccine Revolution
}

\author{
BERNARD DIXON
}

$\mathrm{R}$

arely, if ever, in the annals of science can an ingenious idea have moved so quickly from being an individual, way-out piece of research to being acclaimed as a breakthrough, setting the mold for a whole new generation of health-care products.

It's barely two years since Margaret Liu and her colleagues at the Merck Research Laboratories (West Point, PA) reported that direct injections of a gene from influenza $A$ virus could be used to immunize mice against the disease (Science 259:1745-8, 1993). Yet already, the potential advantages of "DNAvaccines"-ranging from efficacy to cost-have made them the obvious choice for the future. Writing in Parasitology Today (11:113-6, 1993), Gary Waine and Don McManus of the Queensland Institute of Medical Research (Brisbane, Australia) argue that nucleic acids "look set to become the third generation of vaccines."

From the considerable amount of work initiated throughout the world since then, it's clear that many other groups believe Waine and McManus are right. If so, this will mean an innovation as significant as the two other major advances in vaccination that have occurred over the past century. The first was the development by Louis Pasteur and his successors of attenuated and killed forms of microorganisms. The second was the use of defined natural or recombinant components of whole organisms.

But there is additional significance in the Australians' own research. It establishes the utility of naked DNA for immunization, not only against viruses and other organisms that invade host cells, but also against parasites whose life cycle is entirely or predominantly extracellular.

The problem tackled by Liu and her coworkers was the imperfect nature of conventional vaccines against viruses such as influenza $A$, which work by inducing the production of antibodies to proteins on the virus envelope. Those proteins vary from one virus strain to another and change over time. So present-day vaccines afford protection only against those specific antigens-which, of course, were selected before the vaccine was manufactured, on the basis of the previous season's predominant strains. Moreover, these vaccines do not provoke a cytotoxic $\mathrm{T}$ lymphocyte (CTL) response. A vaccine that does so, however, could have the enormous advantage of acting against an internal protein, such as the nucleoprotein (NP), which is conserved in all strains of the virus.
This is what the Merck team achieved. They injected DNA-encoding influenza virus NP into the quadriceps muscle of mice. This induced the synthesis of NP, which in turn provoked the animals to generate CTLs specific for the NP. As a result, the mice were protected not only against the strain of virus from which the NP came but also against another, quite different, strain.

Over the last two years, that single experiment has encouraged many other teams to enter the fray. Their goal: A new generation of vaccines that have all of the advantages and none of the disadvantages of their existing counterparts. Polio and other attenuated vaccines elicit CTL and T helper (Th) cell responses as well as humoral (antibody) immunity. But there is always a possibility, however remote, that the live virus will revert to a dangerous form. On the other hand, both killed and subunit vaccines trigger humoral and Th but not usually CTL responses. The Merck and other studies indicate that DNA vaccines will elicit all three forms of immunity, without the inherent risk associated with live vaccines.

Waine and McManus believe that DNA vaccines (which can be effectively delivered not only by intramuscular injection but also by particle bombardment and by nasal spray) would be significantly cheaper to manufacture than recombinant polypeptides, for example. The considerable costs involved in producing and purifying protein antigens would be avoided, and the DNA could be prepared as a dried pellet for reconstitution immediately before use. This might well obviate the need for a "cold chain" and thus transform the logistics of getting vaccine to remote parts of the world.

There are preliminary indications that DNA vaccines elicit immunity that persists significantly longer than that following conventional immunization. Closely linked with this advantage is an anxiety that the DNA could become integrated into the host genome. This raises possible dangers such as the activation of a host protooncogene or deactivation of a suppressor gene. Waine and McManus describe experiments in which neither this nor the other obvious danger - the induction of anti-DNA antibodies-materialized.

Safety questions still need to be comprehensively answered. But are they really any more formidable than those that have attended the two other primordial developments in vaccine technology over the past century? Quite the reverse, is my verdict. III 